\title{
Thyroid sclerosing mucoepidermoid carcinoma with eosinophilia: a clinicopathologic and molecular analysis of a distinct entity
}

\author{
Akeesha A Shah ${ }^{1}$, Kristin La Fortune ${ }^{2}$, Caitlyn Miller ${ }^{1}$, Stacey E Mills ${ }^{3}$, Zubair Baloch ${ }^{4}$, \\ Virginia LiVolsi ${ }^{4}$, Sanja Dacic ${ }^{1}$, Alyssa L Mahaffey ${ }^{1}$, Marina Nikiforova ${ }^{1}$, Yuri E Nikiforov ${ }^{1}$ \\ and Raja R Seethala ${ }^{1}$ \\ ${ }^{1}$ Department of Pathology, University of Pittsburgh Medical Center, Pittsburgh, PA, USA; ${ }^{2}$ Department of \\ Pathology and Laboratory Medicine, Indiana University Medical Center, Indianapolis, IN, USA; ${ }^{3}$ Department \\ of Pathology, University of Virginia Health Science Center, Charlottesville, VA, USA and ${ }^{4}$ Department of \\ Pathology and Laboratory Medicine, University of Pennsylvania, Philadelphia, PA, USA
}

\begin{abstract}
Sclerosing mucoepidermoid carcinoma with eosinophilia is a rare thyroid neoplasm of uncertain pathogenesis that resembles salivary gland mucoepidermoid carcinoma. This multi-institutional study characterizes the clinicopathologic and molecular features of this tumor by utilizing next-generation sequencing to assess common mutations and gene fusions involved in thyroid carcinogenesis as well as fluorescence in-situ hybridization for MAML2 translocations typical of salivary gland mucoepidermoid carcinoma. Nine cases (6 females and 3 males, mean age: 59 years, range 30-77 years) were identified. All cases were comprised of nests and strands of tumor cells with both squamous and mucinous differentiation embedded in a fibrohyaline stroma with an inflammatory infiltrate replete with eosinophils. All cases were p63 positive, thyroglobulin negative and showed variable expression of TTF-1. All nine cases were negative for MAML2 rearrangements. Five cases successfully tested by next-generation sequencing (ThyroSeq v.2 assay) were negative for mutations and translocations commonly involved in thyroid carcinogenesis. NTRK1 showed overexpression but no evidence of translocation. On follow-up, one patient died of persistent disease, whereas one of four remaining patients with available follow-up (mean: 7.3 years, range 4-11 years) demonstrated recurrence at 4 years. Thus, we show that sclerosing mucoepidermoid carcinoma with eosinophilia appears molecularly and morphologically distinct from follicular and C-cell-derived thyroid tumors as well as from salivary gland mucoepidermoid carcinoma. The overall and recurrence-free survival for these patients may be lower than for other well-differentiated thyroid cancers.
\end{abstract}

Modern Pathology (2017) 30, 329-339; doi:10.1038/modpathol.2016.180; published online 2 December 2016

In 1977, Rhatigan ${ }^{1}$ described a thyroid neoplasm that demonstrated squamous and glandular differentiation, laying the framework for a mucoepidermoid carcinoma category in thyroid, akin to its salivary gland counterpart. Chan et $a l^{2}$ subsequently recognized a variant morphology characterized by characteristic prominent fibrohyaline stroma and an associated eosinophil-rich inflammatory infiltrate, which they designated as sclerosing mucoepidermoid carcinoma with eosinophilia. It has since

Correspondence: Dr AA Shah, Department of Pathology, Robert J. Tomsich Pathology and Laboratory Medicine Institute, Cleveland Clinic, 9500 Euclid Avenue, L-25, Cleveland, OH 44195, USA.

E-mail: AkeeshaAShah@gmail.com

Received 27 June 2016; revised 30 August 2016; accepted 30 August 2016; published online 2 December 2016 become apparent that even within the broad group of thyroid mucoepidermoid carcinoma, sclerosing mucoepidermoid carcinoma with eosinophilia stands out as a distinct entity. Notably, these invariably arise in a background of chronic lymphocytic thyroiditis, and appear to recapitulate the solid cell nest or ultimobranchial body phenotype rather than merely representing squamoglandular metaplasia in a conventional papillary thyroid carcinoma. ${ }^{3-5}$

Given their rarity, the overall clinicopathologic and molecular understanding of sclerosing mucoepidermoid carcinoma with eosinophilia is limited. A recent study has alluded to a potential for aggressive clinical behavior. ${ }^{6}$ Additional key questions of interest include whether these tumors are biologically related to follicular derived thyroid carcinomas, or more to their salivary gland mucoepidermoid 
carcinoma counterparts, particularly with respect to CRCT1/3-MAML2 translocation status. We present the clinicopathologic and molecular findings in nine cases of sclerosing mucoepidermoid carcinoma with eosinophilia and review the literature to expand our understanding of this rare entity.

\section{Materials and methods}

\section{Case Selection}

This study was conducted under approval by the University of Pittsburgh Institutional Review Board (\# PRO1409002). A total of 10 cases of sclerosing mucoepidermoid carcinoma with eosinophilia were retrieved from the consultation and surgical pathology files of three institutions (University of Pittsburgh, University of Pennsylvania and University of Virginia). One of these cases (case \#8) has been previously published. ${ }^{4,7}$ All cases were reviewed by two study authors (RRS and AAS) to confirm their diagnosis and record their clinicopathologic features. The minimum histologic criteria required for a diagnosis of sclerosing mucoepidermoid carcinoma with eosinophilia included: squamous and glandular differentiation, fibrohyaline background and tumor inflammatory infiltrate rich in eosinophils. One case was reviewed by all authors and excluded based on the sparsity of epithelial elements, small size and lack of stromal eosinophilia.

\section{Next-Generation Sequencing}

Mutations and chromosomal rearrangements in genes involved in thyroid carcinogenesis were tested using next-generation sequencing on Ion Torrent Personal Genome Machine (Life Technologies) with ThyroSeq v2 assay as previously described. ${ }^{8}$ In brief, microdissection of tumor-rich areas from unstained slides of formalin-fixed paraffin-embedded tissue was performed under a microscope with hematoxylin and eosin guidance. Two libraries were prepared to study separately point mutations and small indels using $10 \mathrm{ng}$ of isolated DNA, and gene fusions and gene expression controls using $10 \mathrm{ng}$ of isolated RNA. Library concentration and amplicon size were determined using an Agilent BioAnalyzer high sensitivity DNA kit (AgilentTechnologies). Next, multiplexed barcoded libraries were enriched by clonal amplification using emulsion polymerase chain reaction on Ion Sphere particles (IonPGMTemplate OT2 200 kit) and loaded on an Ion 318 Chip. Massive parallel sequencing was carried out on a Personal Genome Machine sequencer (Ion Torrent) using the Ion PGM Sequencing 200 Kit version 2 according to the manufacturer's instructions. This technique detects point mutations and indels in $>1000$ hotspots in 14 thyroid cancer-related genes and 42 gene fusions. ${ }^{8}$ The adequacy of RNA in the tested samples was determined by evaluating the expression of the $P G K$ housekeeping gene.

Additional gene fusions involved in solid tumorigenesis were also assessed using multiplexed polymerase chain reaction amplicon-based nextgeneration sequencing as previously described. ${ }^{9}$ This technique detects 169 known gene fusions involving 19 driver genes: $A K T 3, A L K, B R A F, E G F R$, ERBB4, ERG, FGFR1, FGFR2, FGFR3, MET, NTRK1, NTRK2, NTRK3, NRG1, PDGFRA, PDGFRB, RAF1, RET, ROS1 and 94 partners implicated in solid tumors. The panel also includes amplicons that detect expression in the 5' (extracellular domain) and 3' (tyrosine kinase domain) regions of NTRK1 and other genes, which is used to evaluate whether overexpression of the 3' tyrosine kinase domain is indicative of a novel gene fusion.

\section{Fluorescence In-Situ Hybridization}

Fluorescence in-situ hybridization for MAML2 and NTRK1 gene rearrangements was performed in nine cases. Fluorescence in-situ hybridization for MET amplification was performed in two cases. Positive controls for each of the fluorescence in-situ hybridization studies included salivary gland mucoepidermoid carcinoma with known MAML2 rearrangement, a papillary thyroid carcinoma with known NTRK1 rearrangement and a lung adenocarcinoma with known $C$-MET amplification. Commercially produced break-apart probes for MAML2 11q21 (ZytoLight, Bremerhaven, Germany) and NTRK1 1q23.1 (ZytoLight), as well as $C$-MET (BAC clone RP11-163C9, Empire Genomics, Buffalo, NY, USA) were utilized. Chromosome enumeration probe 7 (Vysis, Abbott Park, IL, USA) was also hybridized together with $C$-MET to serve as an internal control. Slides were deparaffinized and pretreated using the Paraffin Pretreatment Kit (Vysis). Slides were then digested, denatured at $75^{\circ} \mathrm{C}$ for $5 \mathrm{~min}$ in $70 \%$ formamide (Chemicon, Billerica, MA, USA), and successively dehydrated in 70 to $100 \%$ ethanol solutions. In all, $10 \mu \mathrm{l}$ of heat denatured probe was added to the sections, which were then allowed to hybridize overnight in a humidified chamber at $37^{\circ} \mathrm{C}$ (Thermo Fisher Scientific, Inc., Waltham, MA, USA). Post-hybridization washes were carried out and sections were air-dried in the dark and counterstained with DAPI/DAPI-I Antifade (Abbott Molecular, Des Plaines, IL, USA). Sixty non-overlapping nuclei were scored using a Leica fluorescence microscope (Leica DM5500B, Leica Microsystems, Wetzlar, Germany). A case was considered positive for MAML2 or NTRK1 rearrangement if a break-apart signal was identified in $\geq 20 \%$ of tumor nuclei. A case was considered positive for $M E T$ amplification if the ratio of $C-M E T$ copy number to chromosome enumeration probe 7 was $\geq 2$. 
Table 1 Antibodies utilized for immunohistochemistry

\begin{tabular}{lllc}
\hline Antibody & Clone & Company & Dilution \\
\hline Thyroglobulin & Not applicable & Dako & $1: 8000$ \\
P63 & 4A4 & Thermo Fischer & $1: 200$ \\
TTF-1 & 8G7G3/1 & Ventana & Prediluted \\
PAX8 & Not applicable & Proteintech Group & $1: 100$ \\
IgG4 & MRQ-44 & Ventana & $1: 1000$ \\
MET & SP44 & Ventana & Prediluted \\
& & &
\end{tabular}

\section{Immunohistochemistry}

Formalin-fixed paraffin-embedded sections of the specimens were routinely processed and stained with hematoxylin and eosin. Immunohistochemistry for thyroglobulin, p63, TTF-1, PAX8 and IgG4 was performed on $4-\mu$ m-thick sections with commercially purchased antibodies on all cases (Table 1). Immunohistochemistry for MET was also performed on two cases. Automated staining using an indirect, hydrogen peroxide-DAB detection method was performed in a Bond III (Leica Biosystems) and BenchMark ULTRA (Ventana Medical Systems) instruments.

\section{Literature Review}

A literature review was performed in PubMed with search terms (mucoepidermoid carcinoma and thyroid). Cases had to show adequate information to meet the aforementioned minimum histologic criteria applied to the current case cohort to be included in the review. Clinicopathologic features were summarized for all cases.

\section{Results}

\section{Clinicopathologic Features and Follow-Up}

Clinical and staging parameters for our nine sclerosing mucoepidermoid carcinomas with eosinophilia are summarized in Table 2. There was a female predilection of 2:1 with a mean age of 59 (range: 30-77). A thyroid mass (56\%) was the most frequent clinical presentation. Macroscopically, tumors were firm, tan-white to yellow bosselated masses, some with focal cystic change. The mean tumor size was $4.1 \mathrm{~cm}$ (range: $0.5-6.0 \mathrm{~cm}$ ). Lymph node metastasis as well as perineural and angiolymphatic invasion were seen in two patients $(22 \%)$ at the time of presentation; extrathyroidal extension was present in one-third of the patients. Three patients had synchronous or metachronous papillary thyroid carcinomas. In one patient, the papillary thyroid carcinoma was the actual indication for the total thyroidectomy, with the sclerosing mucoepidermoid carcinoma with eosinophilia being an incidental finding. The second patient had a remote history of papillary thyroid carcinoma status-post lobectomy,

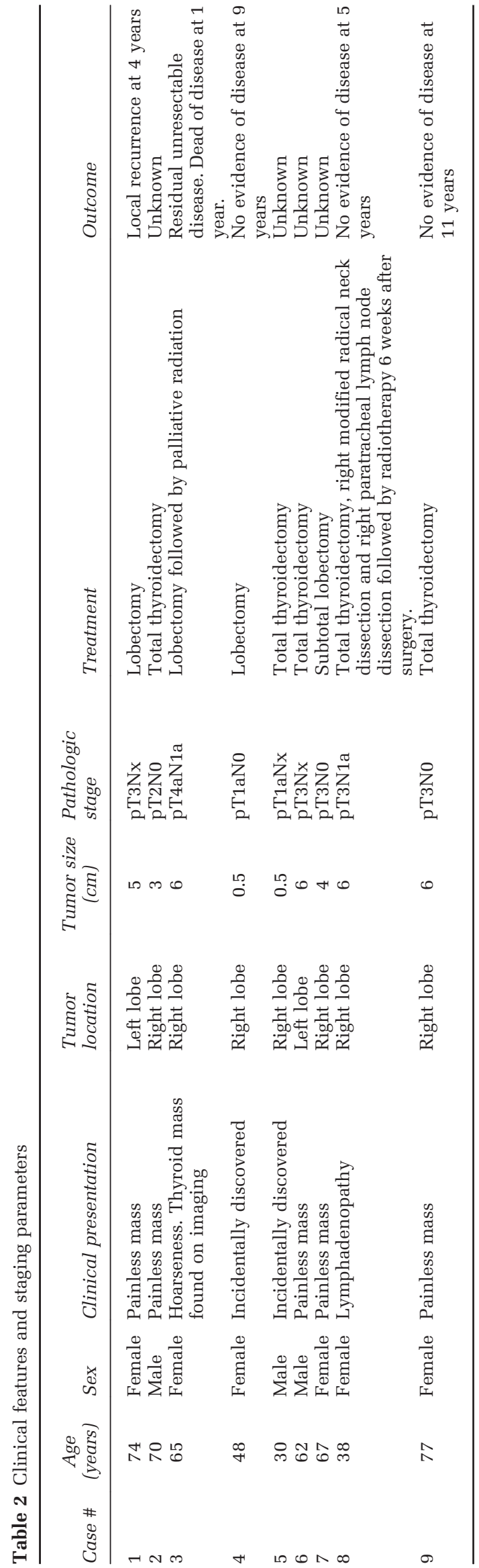



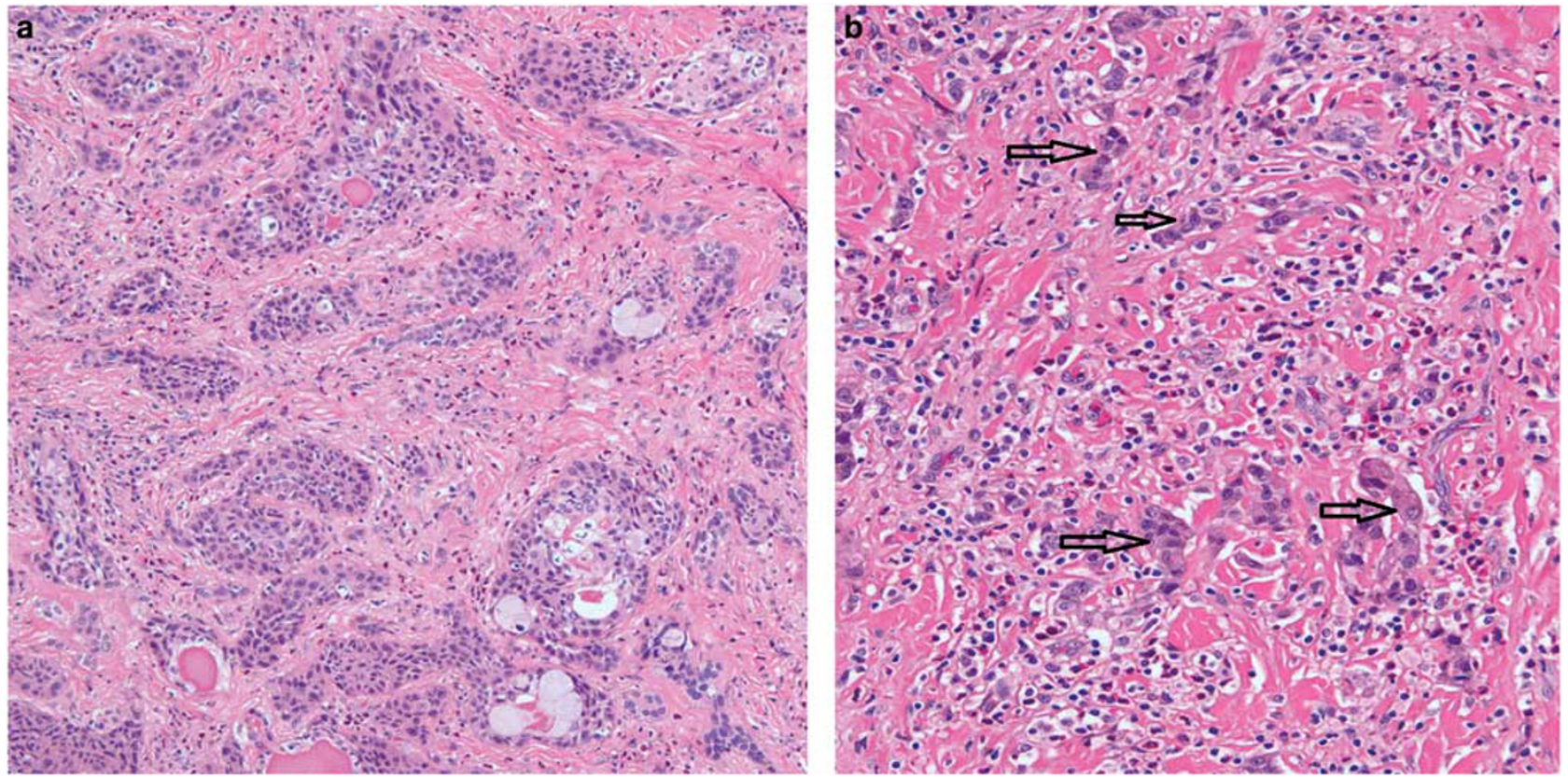

Figure 1 (a) Sclerosing mucoepidermoid carcinoma with eosinophilia composed of variably sized tumor nests within a fibrohyaline stroma that is replete with eosinophils. Scattered goblet cells are also present in the tumor nests. The tumor cells have oval nuclei with prominent nucleoli and eosinophilic cytoplasm. (b) Tumor cells (highlighted with arrows) are occasionally present in thin strands and cords that blend imperceptibly with the background eosinophilic fibrohyaline stroma. However, the eosinophilic infiltrate provides a clue to the presence of tumor cells.

and on surveillance ultrasound was found to have a new mass in her remaining lobe, this was resected and found to be a sclerosing mucoepidermoid carcinoma with eosinophilia. The third patient had a concurrent $0.3 \mathrm{~cm}$ papillary thyroid carcinoma (ie, microcarcinoma).

Microscopically, the tumors consisted of variably sized nests, thin strands and anastomosing cords of lesional cells with squamoglandular differentiation embedded within a fibrohyaline stroma with a background lymphoplasmacytic infiltrate replete with eosinophils (Figures 1a and b). The uninvolved thyroid consistently demonstrated an exuberant chronic lymphocytic thyroiditis. The squamous component was well-developed, showing intercellular bridges, keratohyaline granules and keratin pearl formation (Figure 2a). The glandular component consisted of mucous/goblet cells, which in addition to lining many of the cystic spaces, was also admixed within the squamous component (Figure 2b). The tumor cell nuclei were usually oval in shape with prominent nucleoli and vesicular chromatin. Membrane nuclear irregularities, were common, including small indentations, overt grooves, and rarely, nuclear pseudoinclusions. All but one case showed monomorphic tumor cells with a low mitotic activity (0-2 mitoses/10 high-power fields). Case \#3, however, demonstrated high-grade morphology including nuclear pleomorphism, necrosis, and up to 7 mitoses/10 high-power fields (Figures 2c and d).
Follow-up data were available for five patients. Case \#3 with high-grade morphology showed extrathyroidal extension and invasion of the recurrent laryngeal nerve, larynx and subcutaneous tissue. The patient had persistent disease and died 1 year following diagnosis. Mean follow-up on the other four patients was 7.3 years (range: 4-11). Of these four, one patient (case 1) showed local recurrence in the thyroid bed. None had distant metastasis.

\section{Molecular Profile and Fluorescence In-Situ Hybridization}

Eight of nine cases had sufficient material for nextgeneration sequencing. Of these eight cases, three failed to amplify. The remaining five cases were negative for mutations and gene fusions using the ThyroSeq v2 and solid tumor fusion panel. They showed high expression of the $P G K$ housekeeping gene indicating adequate quality and quantity of RNA, expression of KRT7, and essentially no expression of thyroid follicular cell markers including TG, NIS and TTF1. Five cases showed increased expression of NTRK1 with a slight overexpression of the 3' kinase domain of NTRK1, however, they were negative for NTRK1 fusion tested by the panels. In addition, two cases showed MET overexpression. The additional solid tumor fusion panel, run on the cases with NTRK1 overexpression was negative for 

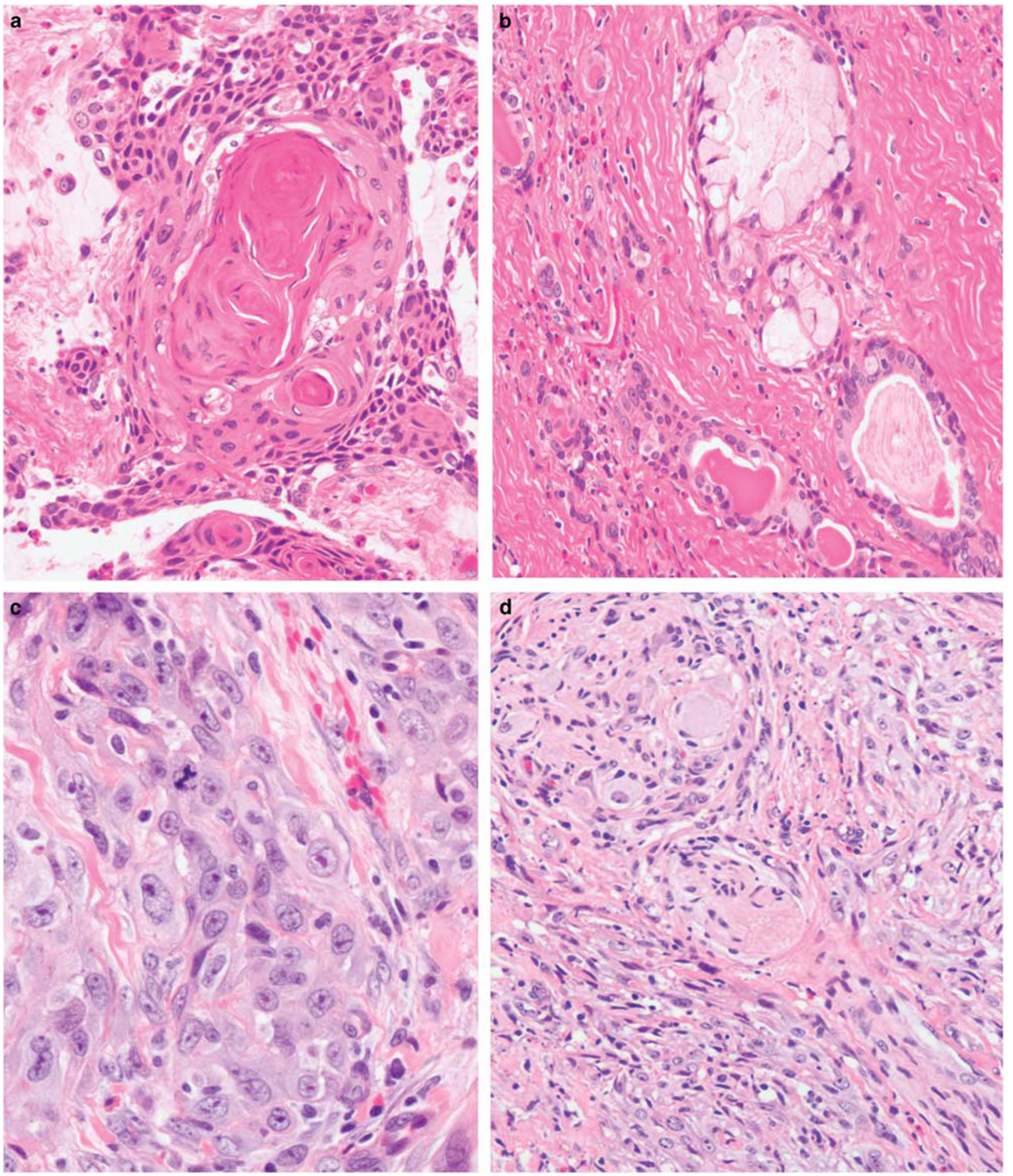

Figure 2 (a) Squamous differentiation in the form of keratin pearls. (b) Mucous cyst formation with goblet cells. (c) Case \#3 with increased nuclear pleomorphism and atypical mitotic figure. (d) Case \#3 with perineural invasion.

NTRK1 gene fusions. To explore the possibility of a novel fusion involving NTRK1, break-apart fluorescence in-situ hybridization was performed on all nine sclerosing mucoepidermoid carcinomas with eosinophilia, however, NTRK1 was intact in all cases (Figure 3a). However, of interest, all cases demonstrated some degree of hyperploidy ranging from 5 to $44 \%$ of tumor cells (mean 20\%). MAML2 by 

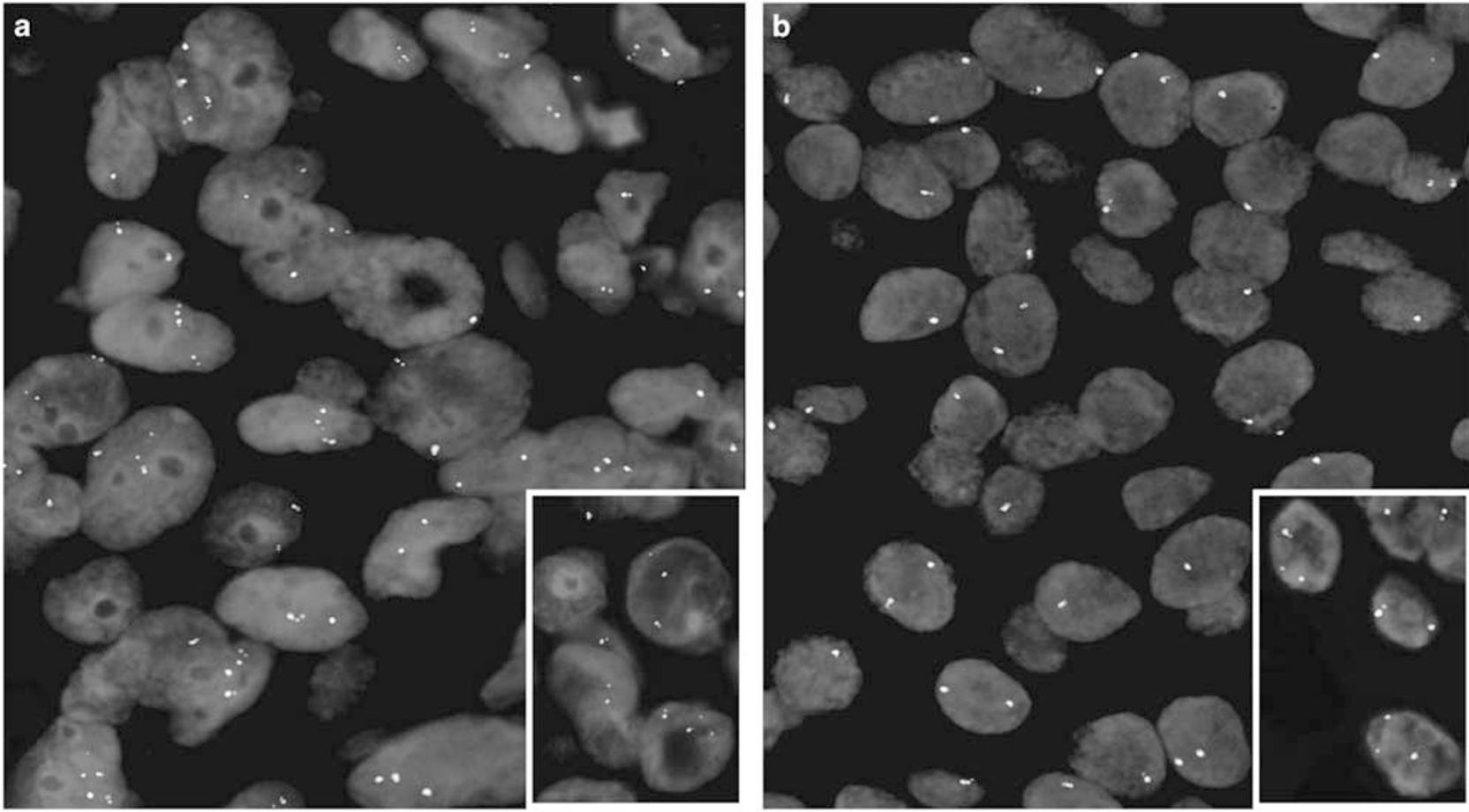

Figure 3 (a) Sclerosing mucoepidermoid carcinoma with eosinophilia with intact NTRK1. Each cell with two intact yellow signals. Inset: positive control (papillary thyroid carcinoma with NTRK1 rearrangement). (b) Sclerosing mucoepidermoid carcinoma with eosinophilia with intact MAML2. Each cell with two intact yellow signals. Inset: positive control (salivary gland mucoepidermoid carcinoma with MAML2 rearrangement). The color reproduction of this figure is available at the Modern Pathology journal online.

fluorescence in-situ hybridization was intact in all nine cases (Figure 3b). The two cases (cases \#1 and \#3) with increased expression of MET was tested for $c-M E T$ amplification by fluorescence in-situ hybridization and were negative, although again both cases showed hyperploidy 12 and 58\% (Figure 4a).

\section{Immunohistochemistry}

All tumors were negative for thyroglobulin and showed diffuse, strong expression of p63 (Figures 5a-c). TTF-1 staining was variable: two cases were completely negative, four cases had weak focal expression in 5-70\% of tumor cells, and three cases had moderate expression in $>75 \%$ of tumor cells (Figures 5e and f). Only one case demonstrated weak PAX8 expression, all other cases were negative for PAX8 (Figure 5g). IgG4 plasma cells ranged from 0 to $12 / 20$ high-power fields (mean $=3.8 / 20$ highpower fields) (Figure 5d). Immunohistochemistry for MET protein expression was performed on the two cases with increased expression of MET by next-generation sequencing and hyperploidy by fluorescence in-situ hybridization. One case was negative, and one case with MET hyperploidy of $58 \%$ demonstrated weak to moderate, focal expression, but not to the extent seen in tumors with amplification by fluorescence in-situ hybridization (Figure 4b).

\section{Literature Review}

Forty-one cases qualified as sclerosing mucoepidermoid carcinoma with eosinophilia through our literature review, ${ }^{2-6,10-22}$ with our series raising the total to 50 cases. The clinicopathologic features for these cases are summarized in Table 3. Overall, the female predilection is even more pronounced as compared with our series ( 13:1). The majority of tumors either occurred in the lateral lobes or diffusely involved the thyroid (98\%) with only one case occurring in the isthmus (1\%). Almost all cases had background chronic lymphocytic thyroiditis (96\%). Extrathyroidal extension and lymph node metastasis were noted at the time of presentation in $47 \%$ and $36 \%$, respectively.

The majority of sclerosing mucoepidermoid carcinomas with eosinophilia were negative $(91 \%)$ for thyroglobulin and all were positive for p63 (100\%) in keeping with a squamous phenotype. TTF-1 expression was more variable with about $64 \%$ of cases demonstrating focal, weak to moderate expression. Only our series examined the expression of PAX8, which was focal and weak in one case and did not parallel TTF-1 expression.

Outcome data for all cases of sclerosing mucoepidermoid carcinoma with eosinophilia in the literature including our nine cases are summarized in Table 4. Follow-up time was either unknown or $<6$ months for 6 cases; the remaining 28 cases had 

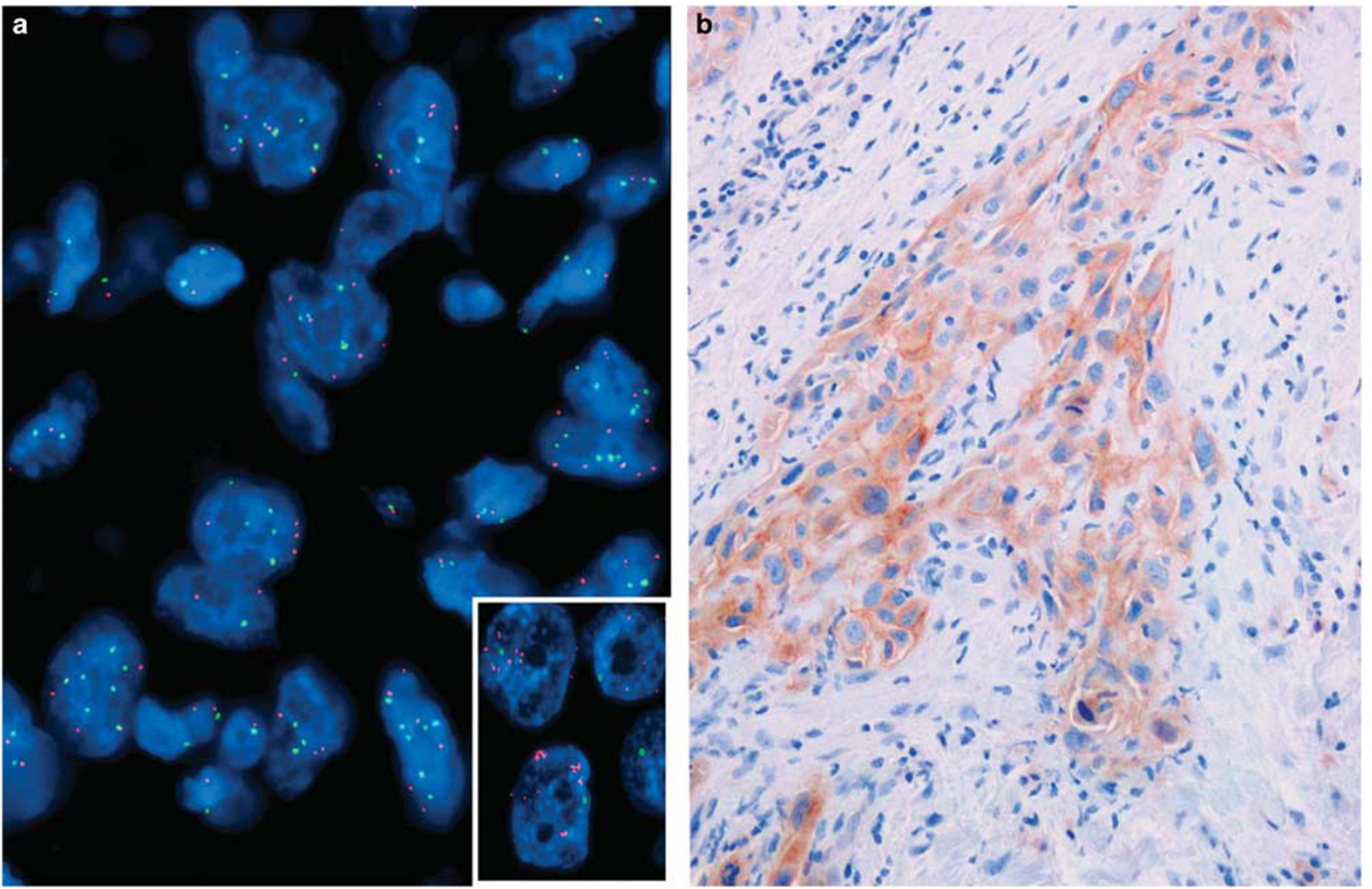

Figure 4 (a) Sclerosing mucoepidermoid carcinoma with eosinophilia without $c$-MET amplification. The ratio of $c$-MET (red) to chromosome enumeration probe 7 (green) is $<2$, however, a fraction of cells show hyperploidy. Inset: positive control (lung adenocarcinoma with $c$-MET amplification). (b) Tumor cells demonstrate focal, weak expression of MET protein by immunohistochemistry.

an average follow-up of 4 years. Following a diagnosis of sclerosing mucoepidermoid carcinoma with eosinophilia, $62 \%$ of patients were alive and free of disease, $15 \%$ were alive with disease and $24 \%$ were deceased either because of their disease or because of an unrelated cause. Data regarding tumor recurrence and metastasis was available in 24 and 28 cases, respectively. The rate of local recurrence was $33 \%$ and with a mean time to recurrence of 2.3 years from the initial diagnosis. The rate of distant metastasis was $29 \%$ with a mean time to metastasis of 2.4 years from the initial diagnosis, with the lung being the most common site. Other sites with documented metastasis include liver, bone and mediastinum.

\section{Discussion}

This study confirms the findings of other authors that sclerosing mucoepidermoid carcinoma with eosinophilia is a rare and still enigmatic thyroid neoplasm of adults that presents on average in the fifth decade. As first noted by Chan et $a l,{ }^{2}$ it has a predilection for women and usually presents as a painless thyroid mass. The histologic features are distinct and consistently noted within our study and in the previously reported cases. As a rule, these tumors occur in a background of chronic lymphocytic thyroiditis, and associated papillary thyroid carcinoma are not uncommon. Surprisingly, about a third of cases show nodal disease, locoregional recurrence and metastasis. This may be perceived as an artifact of reporting bias, or small numbers (50 cases), but suggests that these tumors are more aggressive than previously assumed.

Perhaps the most popular debate about this entity centers around where this tumor sits on the spectrum of thyroid neoplasms. By using standard morphologic and clinicopathologic characteristics, the phenotype of this tumor can be distilled into three candidates for discussion: (1) a unique metaplastic variant of papillary thyroid carcinoma, (2) solid cell nest or ultimobranchial body type neoplasm and (3) ectopic salivary type neoplasm.

The most appealing of these possibilities is a solid cell nest derivation. Like sclerosing mucoepidermoid carcinoma with eosinophilia, solid cell nests demonstrate well-developed squamous differentiation and may contain mucous cells. They are variably cystic and are more prominent in the setting of chronic lymphocytic thyroiditis. The logical 

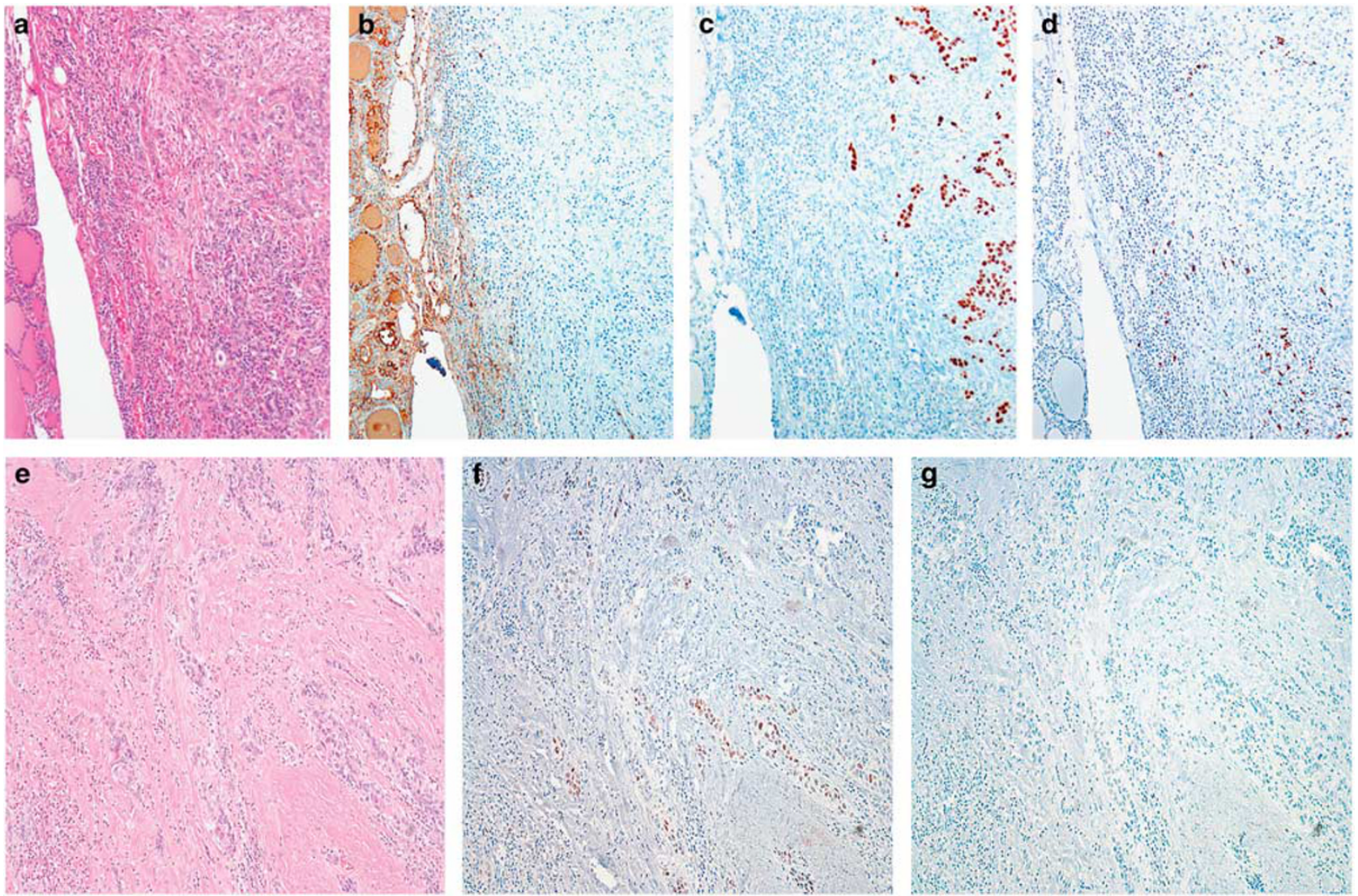

Figure 5 Immunohistochemical staining profile of sclerosing mucoepidermoid carcinoma with eosinophilia. (a) Hematoxylin and eosinstained image for corresponding immunohistochemical stains with tumor cells on right and adjacent normal to left (b). Tumor cells are negative for thyroglobulin. (c) Tumor cells are positive for p63. (d) IgG4-positive plasma cells are scattered throughout the tumor but not significantly increased. (e) Hematoxylin and eosin-stained image for corresponding TTF-1 and PAX8 stains. (f) A subset of tumor cells demonstrate weak to moderate expression of TTF-1. (g) Tumor cells are negative for PAX8.

assumption is that sclerosing mucoepidermoid carcinoma with eosinophilia is malignant transformation of such nests in the setting of chronic lymphocytic thyroiditis. The sparing of the isthmus in terms of subsite distribution mirrors that of the embryologic distribution of solid cell nests (and C cells for that matter). Both solid cell nests and sclerosing mucoepidermoid carcinoma with eosinophilia are p63 positive, essentially thyroglobulin negative and variably TTF-1 positive confirming similarities on an immunophenotypic level. ${ }^{6,16,23}$

Although compelling, this argument is not 'watertight.' Some authors have suggested that solid cell nests are actually metaplastic follicles rather than embryologic remnants. As most thyroid carcinomas are follicular cell derived despite a variety of morphologic appearances, a simple statistical assumption is that sclerosing mucoepidermoid carcinoma with eosinophilia is also part of this spectrum. ${ }^{10,16}$ Similarities in nuclear features have raised the possibility that these are variants of papillary thyroid carcinoma. In fact, primary thyroid mucoepidermoid carcinoma that do not have sclerosis and eosinophilia, and are indeed provisionally categorized as variants and may show a transition to conventional papillary thyroid carcinoma. However, although one-third of our sclerosing mucoepidermoid carcinomas with eosinophilia had preexistent (in the other lobe) or coexistent papillary thyroid carcinoma, no transition between tumor can be documented.

This ongoing debate regarding the origin of sclerosing mucoepidermoid carcinoma with eosinophilia has not been addressed at the molecular level. At present, over $90 \%$ of the driver mutations found in papillary thyroid carcinoma are now known, as a result of the recent multi-platform genome wide surveys, including the cancer genome atlas. ${ }^{24,25}$ Therefore, if sclerosing mucoepidermoid carcinoma with eosinophilia is a variant of papillary thyroid carcinoma, there is a high likelihood that nextgeneration sequencing testing will detect one of these mutations or fusions. However, as expected based on morphologic grounds, none of the known mutations were present in sclerosing mucoepidermoid carcinoma with eosinophilia, further supporting its uniqueness. In addition, none of the cases showed significant $T G$ or TTF1 mRNA expression 
Table 3 Clinical and pathologic features of sclerosing mucoepidermoid carcinomas with eosinophilia in the literature with inclusion of our nine cases

\begin{tabular}{|c|c|}
\hline & $\begin{array}{l}\text { Literature review } \\
\text { and current study }\end{array}$ \\
\hline \multicolumn{2}{|l|}{$\operatorname{Sex}$} \\
\hline Females & $44(88 \%, n=50)$ \\
\hline Males & $6(12 \%, n=50)$ \\
\hline \multicolumn{2}{|l|}{ Age (years) } \\
\hline Mean & 56.1 \\
\hline Range & $30-89$ \\
\hline \multicolumn{2}{|l|}{ Clinical presentation } \\
\hline Painless mass & $36(80 \%, n=45)$ \\
\hline Compressive symptoms & $4(9 \%, n=45)$ \\
\hline Hoarseness & $2(4 \%, n=45)$ \\
\hline Incidental & $2(4 \%, n=45)$ \\
\hline Lymphadenopathy & $1(2 \%, n=45)$ \\
\hline Unknown & 5 \\
\hline \multicolumn{2}{|l|}{ Tumor location } \\
\hline Lateral lobe & $38(88 \%, n=43)$ \\
\hline Isthmus & $1(2 \%, n=43)$ \\
\hline Diffuse involvement & $4(9 \%, n=43)$ \\
\hline Unknown & 7 \\
\hline \multicolumn{2}{|l|}{ Tumor size $(\mathrm{cm})$} \\
\hline Mean & 4.3 \\
\hline Range & $0.5-13$ \\
\hline \multicolumn{2}{|l|}{ Extrathyroidal extension } \\
\hline Present & $21(47 \%, n=45)$ \\
\hline Absent & $24(53 \%, n=45)$ \\
\hline Unknown & 5 \\
\hline $\begin{array}{l}\text { Lymph node metastasis at time of } \\
\text { presentation }\end{array}$ & $18(36 \%, n=50)$ \\
\hline $\begin{array}{l}\text { Synchronous or metachronous papillary } \\
\text { thyroid carcinoma }\end{array}$ & $9(18 \%, n=50)$ \\
\hline \multicolumn{2}{|l|}{ Background chronic lymphocytic thyroiditis } \\
\hline Present & $45(96 \%, n=47)$ \\
\hline Absent & $2(4 \%, n=47)$ \\
\hline Unknown & 3 \\
\hline \multicolumn{2}{|c|}{$\begin{array}{l}\text { Immunohistochemistry \# of cases with positive staining/total \# of } \\
\text { cases }\end{array}$} \\
\hline Thyroglobulin & $4 / 44(9 \%)$ \\
\hline TTF-1 & $11 / 17(65 \%)$ \\
\hline P63 & $19 / 19(100 \%)$ \\
\hline PAX8 & $1 / 9(11 \%)$ \\
\hline \multicolumn{2}{|l|}{ Treatment } \\
\hline Surgery only & $30(65 \%, n=46)$ \\
\hline $\begin{array}{l}\text { Surgery and radiation } \pm \text { chemotherapy or } \\
\text { radioactive iodine }\end{array}$ & $16(35 \%, n=46)$ \\
\hline Unknown & $4(16 \%)$ \\
\hline
\end{tabular}

arguing against a follicular derivation, which is corroborated by the immunohistochemical stains. Despite the absence of definitive molecular alterations, some features were of interest. The overexpression 3' tyrosine kinase domain of NTRK1 is often seen in cases with a translocation involving this gene. However, a more extensive gene fusion panel and fluorescence in-situ hybridization failed to show evidence for this and the mechanism for the
Table 4 Follow-up data of sclerosing mucoepidermoid carcinomas with eosinophilia in the literature with inclusion of our nine cases

\begin{tabular}{|c|c|}
\hline & $\begin{array}{l}\text { Literature review } \\
\text { and current study }\end{array}$ \\
\hline \multicolumn{2}{|c|}{ Patient outcome data available in 34 cases } \\
\hline Alive with no evidence of disease & $21(62 \%)$ \\
\hline Alive with disease & $5(15 \%)$ \\
\hline Total deceased & $8(24 \%)$ \\
\hline Mean time (years) & 4 \\
\hline \multicolumn{2}{|c|}{ Local recurrence data available in 24 cases } \\
\hline Total \# of cases & $8(33 \%)$ \\
\hline Mean time (years) & 2.3 \\
\hline \multicolumn{2}{|l|}{ Site } \\
\hline Soft tissue only & 5 \\
\hline Soft tissue and lymph node & 3 \\
\hline \multicolumn{2}{|c|}{ Distant metastasis data available in 28 cases } \\
\hline Total \# of cases & $8(29 \%)$ \\
\hline At time of initial diagnosis & 1 (lung, spinal cord) \\
\hline Subsequent to initial diagnosis & 7 \\
\hline \multicolumn{2}{|l|}{ Site } \\
\hline Lung & 4 \\
\hline Lung and bone & 1 \\
\hline Liver & 1 \\
\hline Other & 1 (mediastinum) \\
\hline
\end{tabular}

overexpression is still unclear. Similarly, a few cases showed MET overexpression but no amplification by fluorescence in-situ hybridization, although one case with a high percentage of MET hyperploidy did express MET protein immunohistochemically.

The third hypothesis that sclerosing mucoepidermoid carcinoma with eosinophilia is a salivary type mucoepidermoid carcinoma, possibly arising from ectopic salivary rests was also considered. Of note, a form of 'sclerosing mucoepidermoid carcinoma with eosinophilia' has been described in the parotid gland. ${ }^{26-28}$ It is known that the vast majority of salivary gland mucoepidermoid carcinoma harbor a CRTC1 or CRTC3-MAML2 translocation, regardless of morphology. ${ }^{29-32}$ In addition, a subset of sclerosing mucoepidermoid carcinoma of the salivary gland demonstrate an increased IgG4 plasma cell fraction. ${ }^{33,34}$ However, all cases were negative by break-apart MAML2 FISH. Furthermore, although there were small IgG4-positive plasma cell components in sclerosing mucoepidermoid carcinoma with eosinophilia, they were not significant enough to invoke IgG4 spectrum of disease. Interestingly, when morphologic features are scrutinized in detail, thyroid sclerosing mucoepidermoid carcinoma with eosinophilia is similar but still different from salivary gland mucoepidermoid carcinoma. Unlike salivary gland mucoepidermoid carcinoma, mature squamous differentiation (ie, keratinization) is a common and even defining feature of sclerosing mucoepidermoid carcinoma with eosinophilia. 
Furthermore, the intermediate type cells that define salivary gland mucoepidermoid carcinoma are not present in sclerosing mucoepidermoid carcinoma with eosinophilia of thyroid.

The lack of a defining molecular alteration in sclerosing mucoepidermoid carcinoma with eosinophilia is disappointing, but not unexpected, as sclerosing mucoepidermoid carcinoma with eosinophilia is already favored to be morphologically distinct from papillary thyroid carcinoma and will consequently lack any of its associated mutations. However, given the current trends in solid tumors, it would not be surprising that sclerosing mucoepidermoid carcinoma with eosinophilia, a tumor with a distinct morphology, will ultimately be characterized by a reproducible molecular alteration with more in-depth whole exome studies. A secondary question arises as to the status of the other thyroid mucoepidermoid carcinomas, that is, those without sclerosis or eosinophilia: are they also distinct from papillary thyroid carcinoma and are they more related to sclerosing mucoepidermoid carcinoma with eosinophilia? As we did not have enough of these mucoepidermoid carcinomas for a formal comparison, this will remain unknown for some time. However, we did encounter one mucoepidermoid carcinoma that demonstrated conventional foci of papillary thyroid carcinoma and a RET translocation using an earlier version of ThyroSeq (data unpublished).

In summary, thyroid sclerosing mucoepidermoid carcinoma with eosinophilia is a morphologically and biologically distinct entity, and does not harbor mutations commonly detected in thyroid carcinomas. Thyroid sclerosing mucoepidermoid carcinoma with eosinophilia is also consistently MAML2 negative arguing against a relationship to salivary gland mucoepidermoid carcinoma. This absence of molecular alterations indirectly adds further support for a solid cell nest phenotype for these tumors. Sclerosing mucoepidermoid carcinoma with eosinophilia are usually low grade, but have more potential for aggressive clinical behavior than initially thought.

\section{Disclosure/conflict of interest}

The authors declare no conflict of interest.

\section{References}

1 Rhatigan RM, Roque JL, Bucher RL Mucoepidermoid carcinoma of the thyroid gland. Cancer 1977;39: 210-214.

2 Chan JK, Albores-Saavedra J, Battifora H, et al. Sclerosing mucoepidermoid thyroid carcinoma with eosinophilia. A distinctive low-grade malignancy arising from the metaplastic follicles of Hashimoto's thyroiditis. Am J Surg Pathol 1991;15:438-448.

3 Sim SJ, Ro JY, Ordonez NG, et al. Sclerosing mucoepidermoid carcinoma with eosinophilia of the thyroid: report of two patients, one with distant metastasis, and review of the literature. Hum Pathol 1997;28:1091-1096.

4 Baloch ZW, Solomon AC, LiVolsi VA. Primary mucoepidermoid carcinoma and sclerosing mucoepidermoid carcinoma with eosinophilia of the thyroid gland: a report of nine cases. Mod Pathol 2000;13: 802-807.

5 Hunt JL, LiVolsi VA, Barnes EL. p63 expression in sclerosing mucoepidermoid carcinomas with eosinophilia arising in the thyroid. Mod Pathol 2004;17: 526-529.

6 Quiroga-Garza G, Lee JH, El-Naggar A, et al. Sclerosing mucoepidermoid carcinoma with eosinophilia of the thyroid: more aggressive than previously reported. Hum Pathol 2015;46:725-731.

7 Solomon AC, Baloch ZW, Salhany KE, et al. Thyroid sclerosing mucoepidermoid carcinoma with eosinophilia: mimic of Hodgkin disease in nodal metastases. Arch Pathol Lab Med 2000;124:446-449.

8 Nikiforov YE, Carty SE, Chiosea SI, et al. Highly accurate diagnosis of cancer in thyroid nodules with follicular neoplasm/suspicious for a follicular neoplasm cytology by ThyroSeq v2 next-generation sequencing assay. Cancer 2014;120:3627-3634.

9 Beadling C, Wald AI, Warrick A, et al. A multiplexed amplicon approach for detecting gene fusions by nextgeneration sequencing. J Mol Diagn 2016;18:165-175.

10 Wenig BM, Adair CF, Heffess CS. Primary mucoepidermoid carcinoma of the thyroid gland: a report of six cases and a review of the literature of a follicular epithelial-derived tumor. Hum Pathol 1995;26: 1099-1108.

11 Bondeson L, Bondeson AG. Cytologic features in fineneedle aspirates from a sclerosing mucoepidermoid thyroid carcinoma with eosinophilia. Diagn Cytopathol 1996;15:301-305.

12 Geisinger KR, Steffee CH, McGee RS, et al. The cytomorphologic features of sclerosing mucoepidermoid carcinoma of the thyroid gland with eosinophilia. Am J Clin Pathol 1998;109:294-301.

13 Chung J, Lee SK, Gong G, et al. Sclerosing mucoepidermoid carcinoma with eosinophilia of the thyroid glands: a case report with clinical manifestation of recurrent neck mass. J Korean Med Sci 1999;14: $338-341$.

14 Cameselle-Teijeiro J, Febles-Perez C, Sobrinho-Simoes M. Papillary and mucoepidermoid carcinoma of the thyroid with anaplastic transformation: a case report with histologic and immunohistochemical findings that support a provocative histogenetic hypothesis. Patho Res Pract 1995;191:1214-1221.

15 Sharma K, Nigam S, Khurana N, et al. Sclerosing mucoepidermoid carcinoma with eosinophilia of the thyroid-a case report. Indian J Pathol Microbiol 2003;46:660-661.

16 Albores-Saavedra J, Gu X, Luna MA. Clear cells and thyroid transcription factor I reactivity in sclerosing mucoepidermoid carcinoma of the thyroid gland. Ann Diagn Pathol 2003;7:348-353.

17 Shehadeh NJ, Vernick J, Lonardo F, et al. Sclerosing mucoepidermoid carcinoma with eosinophilia of the thyroid: a case report and review of the literature. Am J Otolaryngol 2004;25:48-53.

18 Cavazza A, Toschi E, Valcavi R, et al. [Sclerosing mucoepidermoid carcinoma with eosinophilia of the thyroid: description of a case]. Pathologica 1999;91: 31-35. 
19 Das S, Kalyani R. Sclerosing mucoepidermoid carcinoma with eosinophilia of the thyroid. Indian J Pathol Microbiol 2008;51:34-36.

20 Frazier WD, Patel NP, Sullivan CA. Pathology quiz case 1. Sclerosing mucoepidermoid carcinoma with eosinophilia (SMECE). Arch Otolaryngol Head Neck Surg 2008;134:5

21 Calo PG, Maxia S, Lai ML, et al. Sclerosing mucoepidermoid thyroid carcinoma requiring cervical reconstruction: a case report and review of the literature. Am Surg 2010;76:918-919.

22 Lai CY, Chao TC, Lin JD, et al. Sclerosing mucoepidermoid carcinoma with eosinophilia of thyroid gland in a male patient: a case report and literature review. Int J Clin Exp Pathol 2015;8:5947-5951.

23 Asioli S, Erickson LA, Lloyd RV. Solid cell nests in Hashimoto's thyroiditis sharing features with papillary thyroid microcarcinoma. Endocr Pathol 2009;20: 197-203.

24 Hsiao SJ, Nikiforov YE. Molecular approaches to thyroid cancer diagnosis. Endocr Relat Cancer 2014;21: T301-T313.

25 Cancer Genome Atlas Research N. Integrated genomic characterization of papillary thyroid carcinoma. Cell 2014;159:676-690.

26 Chan JK, Saw D. Sclerosing mucoepidermoid tumour of the parotid gland: report of a case. Histopathology 1987;11:203-207.
27 Muller S, Barnes L, Goodurn WJ Jr. Sclerosing mucoepidermoid carcinoma of the parotid. Oral Surg Oral Med Oral Pathol Oral Radiol Endod 1997;83:685-690.

28 Urano M, Abe M, Horibe Y, et al. Sclerosing mucoepidermoid carcinoma with eosinophilia of the salivary glands. Pathol Res Pract 2002;198:305-310.

29 Seethala RR, Dacic S, Cieply K, et al. A reappraisal of the MECT1/MAML2 translocation in salivary mucoepidermoid carcinomas. Am J Surg Pathol 2010;34: 1106-1121.

30 Behboudi A, Enlund F, Winnes M, et al. Molecular classification of mucoepidermoid carcinomas-prognostic significance of the MECT1-MAML2 fusion oncogene. Genes Chromosomes Cancer 2006;45:470-481.

31 Fehr A, Roser K, Heidorn K, et al. A new type of MAML2 fusion in mucoepidermoid carcinoma. Genes Chromosomes Cancer 2008;47:203-206.

32 Martins C, Cavaco B, Tonon G, et al. A study of MECT1-MAML2 in mucoepidermoid carcinoma and Warthin's tumor of salivary glands. J Mol Diagn 2004;6: 205-210.

33 Tian W, Yakirevich E, Matoso A, et al. IgG4(+) plasma cells in sclerosing variant of mucoepidermoid carcinoma. Am J Surg Pathol 2012;36:973-979.

34 Tasaki T, Matsuyama A, Tabata T, et al. Sclerosing mucoepidermoid carcinoma with eosinophilia of the salivary gland: case report and review of the literature. Pathol Int 2013;63:125-131. 